EPJ Web of Conferences 49, 03002 (2013)

DOI: $10.1051 /$ epjconf/20134903002

(C) Owned by the authors, published by EDP Sciences, 2013

\title{
Selection of LHCb Physics Results
}

\author{
Burkhard Schmidt ${ }^{1, \mathrm{a}}$, on behalf of the LHCb collaboration. \\ ${ }^{1}$ CERN, Geneva, Switzerland
}

\begin{abstract}
LHCb is a dedicated flavour physics experiment at the LHC searching for physics beyond the Standard Model through precision measurements of $C P$-violating observables and the study of very rare decays of beauty- and charm-flavoured hadrons. In this article a selection of recent LHCb results is presented. Unless otherwise stated, the results are based on an integrated luminosity of $1 \mathrm{fb}^{-1}$ accumulated during the year 2011 at $\sqrt{s}=7 \mathrm{TeV}$.
\end{abstract}

\section{Introduction}

The LHCb detector has been taking data with high efficiency during the first three years of LHC operation, producing a wealth of exciting physics results, which have made an impact on the flavour physics landscape and proved the concept of a dedicated heavy flavour experiment in the forward region of a hadron collider. Details about the LHCb performance during this time are summarized in a separate contribution [1].

The LHC is an intense source of $b$ hadrons. The $b \bar{b}$ cross-section in proton-proton collisions at $\sqrt{s}=7 \mathrm{TeV}$ is about $300 \mu \mathrm{b}$, implying that more than $10^{11} b \bar{b}$ pairs are produced per $\mathrm{fb}^{-1}$. The $c \bar{c}$ cross-section is about 20 times larger than the $b \bar{b}$ cross-section, giving LHCb great potential in charm physics studies as well.

This article focusses on some important physics results presented at the HCP Symposium 2012, which are related to three areas of research carried out by the LHCb collaboration: $C P$ violation measurements, rare decays and charm physics. In Section 2 a measurement of the CKM unitary triangle phase $\gamma$ at tree level is presented, Section 3 summarizes searches of some rare decays to muonic final states, and Section 4 reports about the measurement of $D^{0}-\bar{D}^{0}$ mixing.

\section{Measuring the $C P$-violating phase $\gamma$}

$C P$ violation phenomena in the Standard Model (SM) are the result of a single phase in the CKM quark-mixing matrix $[2,3]$. The unitarity condition formed from the CKM matrix elements, $V_{u d} V_{u b}^{*}+V_{c d} V_{c b}^{*}+V_{t d} V_{t b}^{*}=0$, describes a triangle in the complex plane. The least-well determined observable, the phase $\gamma=\arg \left(-V_{u d} V_{u b}^{*} / V_{c d} V_{c b}^{*}\right)$ is of particular interest as $\gamma \neq 0$ can produce direct $C P$ violation in tree-level decays. To date, most direct information on $\gamma$ is derived from the $B$ factory and Tevatron measurements.
These results must be combined though to form final conclusions. The direct determination of $\gamma$ via fits to the experimental data gives $(66 \pm 12)^{\circ}[4]$ or $(76 \pm 10)^{\circ}$ [5], depending on whether a frequentist or Bayesian treatment is used.

A measurement of the partial widths of $B^{ \pm} \rightarrow D K^{ \pm}$decays, where the $D$ signifies a $D^{0}$ or $\bar{D}^{0}$ meson, is one of the most powerful experimental methods of determining $\gamma$. If the $D$ final state is accessible for both $D^{0}$ and $\bar{D}^{0}$ mesons, the interference of these two processes provides sensitivity to $\gamma$ and may exhibit direct $C P$ violation. This feature of open-charm $B$-decays was first recognised in its application to $C P$ eigenstates, such as $D \rightarrow K^{+} K^{-}, \pi^{+} \pi^{-}[6,7]$, but can be extended to other decays, e.g. $D \rightarrow \pi^{-} K^{+}$, known as "ADS" modes in reference to the authors $[8,9]$.

Self $C P$-conjugate three-body decays may also be used, such as $K_{S}^{0} \pi^{+} \pi^{-}$and $K_{S}^{0} K^{+} K^{-}$, designated collectively as $K_{S}^{0} h^{+} h^{-}$[10]. The strategy relies on comparing the distribution of events in the $D \rightarrow K_{s}^{0} h^{+} h^{-}$Dalitz plot for $B^{ \pm} \rightarrow D K^{ \pm}$decays. However, in order to determine $\gamma$ it is necessary to know how the strong phase of the $D$ decay varies over the Dalitz plot. While one approach is to use an amplitude model fitted on flavour-tagged $D \rightarrow K_{S}^{0} h^{+} h^{-}$ decays to provide this input [11-16], a novel approach is to make use of direct measurements of the strong phase behaviour in bins of the Dalitz plot, which may be obtained from quantum-correlated $D \bar{D}$ pairs from $\psi(3770)$ decays, thereby avoiding the need to assign a model-related systematic uncertainty. These $\psi(3770)$ decays are available from CLEO-c [17]. A first model-independent analysis was recently presented by Belle [18] using $B^{ \pm} \rightarrow D K^{ \pm}$, $D \rightarrow K_{S}^{0} \pi^{+} \pi^{-}$decays.

\subsection{2 -body $D^{0}$ decays}

The amplitude of the decay $B^{+} \rightarrow D K^{+}$can be written as the superposition of the $B^{+} \rightarrow \bar{D}^{0} K^{+}$and $B^{+} \rightarrow D^{0} K^{+}$ contributions as

$$
A_{B}=\bar{A}+r_{B} e^{i\left(\delta_{B}+\gamma\right)} A
$$

ae-mail: Burkhard.Schmidt@cern.ch

$$
A_{B}=\bar{A}+r_{B} e^{i\left(\delta_{B}+\gamma\right)} A
$$

(n) 
Here $A$ is the $D^{0}$ decay amplitude, and and $\bar{A}$ the $\bar{D}^{0}$ amplitude. The parameter $r_{B}$, the ratio of the magnitudes of the $B^{+} \rightarrow D^{0} K^{+}$and $B^{+} \rightarrow \bar{D}^{0} K^{+}$amplitudes, is $\sim 0.1$ [19], and $\delta_{B}$ is the strong phase difference between them. The equivalent expression for the charge-conjugated decay $B^{-} \rightarrow D K^{-}$is obtained by making the substitutions $\gamma \rightarrow-\gamma$ and $A \leftrightarrow \bar{A}$. The CKM phase $\gamma$ can be measured from the observed rates of the possible $B \rightarrow D K$ decays.

We studied $B^{ \pm}$decays in the $C P$ modes, $\left[K^{+} K^{-}\right]_{D} h^{ \pm}$ and $\left[\pi^{+} \pi^{-}\right]_{D} h^{ \pm}$, the suppressed ADS mode $\left[\pi^{ \pm} K^{\mp}\right]_{D} h^{ \pm}$and the favoured $\left[K^{ \pm} \pi^{\mp}\right]_{D} h^{ \pm}$combination, where $h$ indicates either a $\pi$ or a $K . B^{-} \rightarrow D \pi^{-}$decays have lesser sensitivity to $\gamma$, but provide a high-statistics control sample from which probability density functions (PDFs) are shaped. In total 13 observables are measured: three ratios of partial widths

$$
R_{K / \pi}^{f}=\frac{\Gamma\left(B^{-} \rightarrow[f]_{D} K^{-}\right)+\Gamma\left(B^{+} \rightarrow[f]_{D} K^{+}\right)}{\Gamma\left(B^{-} \rightarrow[f]_{D} \pi^{-}\right)+\Gamma\left(B^{+} \rightarrow[f]_{D} \pi^{+}\right)},
$$

where $f$ represents $K K, \pi \pi$ and the favoured $K \pi$ mode, six $C P$ asymmetries

$$
A_{h}^{f}=\frac{\Gamma\left(B^{-} \rightarrow[f]_{D} h^{-}\right)-\Gamma\left(B^{+} \rightarrow[f]_{D} h^{+}\right)}{\Gamma\left(B^{-} \rightarrow[f]_{D} h^{-}\right)+\Gamma\left(B^{+} \rightarrow[f]_{D} h^{+}\right)},
$$

and four charge-separated partial widths of the ADS mode relative to the favoured mode

$$
R_{h}^{ \pm}=\frac{\Gamma\left(B^{ \pm} \rightarrow\left[\pi^{ \pm} K^{\mp}\right]_{D} h^{ \pm}\right)}{\Gamma\left(B^{ \pm} \rightarrow\left[K^{ \pm} \pi^{\mp}\right]_{D} h^{ \pm}\right)} .
$$

From the $1 \mathrm{fb}^{-1}$ of data collected in 2011 an analysis has been performed to measure these observables. The following quantities can be deduced from them:

$$
\begin{aligned}
R_{C P+} & \approx<R_{K / \pi}^{K K}, R_{K / \pi}^{\pi \pi}>/ R_{K / \pi}^{K \pi} \\
& =1.007 \pm 0.038 \pm 0.012 \\
A_{C P+} & =<A_{K}^{K K}, A_{K}^{\pi \pi}> \\
& =0.145 \pm 0.032 \pm 0.010 \\
R_{\mathrm{ADS}(K)} & =\left(R_{K}^{-}+R_{K}^{+}\right) / 2 \\
& =0.0152 \pm 0.0020 \pm 0.0004 \\
A_{\mathrm{ADS}(K)} & =\left(R_{K}^{-}-R_{K}^{+}\right) /\left(R_{K}^{-}+R_{K}^{+}\right) \\
& =-0.52 \pm 0.15 \pm 0.02 \\
R_{\mathrm{ADS}(\pi)} & =\left(R_{\pi}^{-}+R_{\pi}^{+}\right) / 2 \\
& =0.00410 \pm 0.00025 \pm 0.00005 \\
A_{\mathrm{ADS}(\pi)} & =\left(R_{\pi}^{-}-R_{\pi}^{+}\right) /\left(R_{\pi}^{-}+R_{\pi}^{+}\right) \\
& =0.143 \pm 0.062 \pm 0.011 .
\end{aligned}
$$

The correlations between systematic uncertainties have been taken into account in the combination and angled brackets indicate weighted averages.

From these measurements, we observe direct $C P$ violation in $B^{ \pm} \rightarrow D K^{ \pm}$decays with a total significance of $5.8 \sigma$. Further details on this analysis can be found in Ref. [20]. These results contain dependence on the weak phase $\gamma$ and form an important contribution to the measurement of this parameter, as outlined in Section 2.4.

\subsection{3-body $D^{0}$ decays}

The amplitude of the decay $B^{+} \rightarrow D K^{+}, D \rightarrow K_{S}^{0} h^{+} h^{-}$ can be written as Eq. 1, except that now $A_{B}$ depends on $m_{+}^{2}$ and $m_{-}^{2}$, the invariant masses squared of the $K_{s}^{0} h^{+}$ and $K_{s}^{0} h^{-}$combinations, respectively, that define the position of the decay in the Dalitz plot. $A=A\left(m_{+}^{2}, m_{-}^{2}\right)$ is the $D^{0} \rightarrow K_{s}^{0} h^{+} h^{-}$amplitude, and $\bar{A}=\bar{A}\left(m_{+}^{2}, m_{-}^{2}\right)$ the $\bar{D}^{0} \rightarrow K_{s}^{0} h^{+} h^{-}$amplitude. Neglecting $C P$ violation, which is known to be small in $D^{0}-\bar{D}^{0}$ mixing and Cabibbofavoured $D$ meson decays [21], the conjugate amplitudes are related by $A\left(m_{+}^{2}, m_{-}^{2}\right)=\bar{A}\left(m_{-}^{2}, m_{+}^{2}\right)$.

Following the formalism set out in Ref. [10], the Dalitz plot is partitioned into $2 \mathrm{~N}$ regions symmetric under the exchange $m_{+}^{2} \leftrightarrow m_{-}^{2}$. The bins are labelled from $-N$ to $+N$ (excluding zero), where the positive bins satisfy $m_{-}^{2}>m_{+}^{2}$. At each point in the Dalitz plot, there is a strong-phase difference $\delta_{D}\left(m_{+}^{2}, m_{-}^{2}\right)=\arg \bar{A}-\arg A$ between the $\bar{D}^{0}$ and $D^{0}$ decay. The cosine of the strong-phase difference averaged in each bin and weighted by the absolute decay rate is termed $c_{i}$ and is given by

$$
c_{i}=\frac{\int_{\mathcal{D}_{i}}\left(|A||\bar{A}| \cos \delta_{D}\right) d \mathcal{D}}{\sqrt{\int_{\mathcal{D}_{i}}|A|^{2} d \mathcal{D}} \sqrt{\int_{\mathcal{D}_{i}}|\bar{A}|^{2} d \mathcal{D}}},
$$

where the integrals are evaluated over the area $\mathcal{D}$ of bin $i$. An analogous expression may be written for $s_{i}$, which is the sine of the strong-phase difference within bin $i$, weighted by the decay rate. Direct measurements of $c_{i}$ and $s_{i}$ have been performed at CLEO-c, exploiting quantumcorrelated $D \bar{D}$ pairs produced at the $\psi(3770)$ resonance.

The population of each positive (negative) bin in the Dalitz plot arising from $B^{+}$decays is $N_{+i}^{+}\left(N_{-i}^{+}\right)$, and that from $B^{-}$decays is $N_{+i}^{-}\left(N_{-i}^{-}\right)$. From Eq. 1 it follows that

$N_{ \pm i}^{+}=h_{B^{+}}\left[K_{\mp i}+\left(x_{+}^{2}+y_{+}^{2}\right) K_{ \pm i}+2 \sqrt{K_{i} K_{-i}}\left(x_{+} c_{ \pm i} \mp y_{+} s_{ \pm i}\right)\right]$, $N_{ \pm i}^{-}=h_{B^{-}}\left[K_{ \pm i}+\left(x_{-}^{2}+y_{-}^{2}\right) K_{\mp i}+2 \sqrt{K_{i} K_{-i}}\left(x_{-} c_{ \pm i} \pm y_{-} s_{ \pm i}\right)\right]$, where $h_{B^{ \pm}}$are normalisation factors which can, in principle, be different for $B^{+}$and $B^{-}$due to the production asymmetries, and $K_{i}$ is the number of events in bin $i$ of the decay of a flavour tagged $D^{0} \rightarrow K_{S}^{0} h^{+} h^{-}$Dalitz plot. The sensitivity to $\gamma$ enters through the Cartesian parameters

$$
x_{ \pm}=r_{B} \cos \left(\delta_{B} \pm \gamma\right) \text { and } y_{ \pm}=r_{B} \sin \left(\delta_{B} \pm \gamma\right) .
$$

The CLEO-c study segments the $K_{s}^{0} \pi^{+} \pi^{-}$Dalitz plot into $2 \times 8$ bins. Several bin definitions are available; we adopted the 'optimal binning' variant. For the $K_{\mathrm{s}}^{0} K^{+} K^{-}$ final state we used measurements for the Dalitz plot partitioned into $2 \times 2$ bins. Finer binning was available for this mode, but was not used due to the size of our signal sample.

Approximately $800 B^{ \pm} \rightarrow D K^{ \pm}$decay candidates, with the $D$ meson decaying either to $K_{s}^{0} \pi^{+} \pi^{-}$or $K_{s}^{0} K^{+} K^{-}$, have been selected from $1.0 \mathrm{fb}^{-1}$ of data collected by LHCb in 2011. In this analysis the observed distribution of candidates over the $D^{0} \rightarrow K_{s}^{0} h^{+} h^{-}$Dalitz plot is used to determine the $C P$-violating parameters $x_{ \pm}, y_{ \pm}$and $h_{B^{ \pm}}$. An uncertainty is assigned that is related to the precision of the 
$c_{i}$ and $s_{i}$ measurements. When interpreting these results in terms of the underlying physics parameters it is found that $r_{B}=0.07 \pm 0.04, \gamma=\left(44_{-38}^{+43}\right)^{\circ}$ and $\delta_{B}=\left(137_{-46}^{+35}\right)^{\circ}$. These values are consistent with the world average of results from previous measurements [21], although the uncertainties on $\gamma$ and $\delta_{B}$ are large. This is partly driven by the relatively low central value that is obtained for the parameter $r_{B}$. Further details are provided in Ref. [22].

\subsection{4-body $D^{0}$ decays}

ADS observables similar to those discussed in Section 2.1 have been measured at $\mathrm{LHCb}$ using $B^{ \pm} \rightarrow D h^{ \pm}$decays, where the $D$ meson is reconstructed in the four-body final state $K^{ \pm} \pi^{\mp} \pi^{+} \pi^{-}$. From these measurements the following quantities can be deduced:

$$
\begin{aligned}
R_{\mathrm{ADS}(K)}^{K 3 \pi} & =\left(R_{K}^{K 3 \pi,-}+R_{K}^{K 3 \pi,+}\right) / 2 \\
& =0.0124 \pm 0.0027 \\
A_{\mathrm{ADS}(K)}^{K 3 \pi} & =\left(R_{K}^{K 3 \pi,-}-R_{K}^{K 3 \pi,+}\right) /\left(R_{K}^{K 3 \pi,-}+R_{K}^{K 3 \pi,+}\right) \\
& =-0.42 \pm 0.22 \\
R_{\mathrm{ADS}(\pi)}^{K 3 \pi} & =\left(R_{\pi}^{K 3 \pi,-}+R_{\pi}^{K 3 \pi,+}\right) / 2 \\
& =0.00369 \pm 0.00036 \\
A_{\mathrm{ADS}(\pi)}^{K 3 \pi} & =\left(R_{\pi}^{K 3 \pi,-}-R_{\pi}^{K 3 \pi,+}\right) /\left(R_{\pi}^{K 3 \pi,-}+R_{\pi}^{K 3 \pi,+}\right) \\
& =+0.13 \pm 0.10,
\end{aligned}
$$

We observed for the first time the suppressed ADS modes $B^{ \pm} \rightarrow\left[\pi^{ \pm} K^{\mp} \pi^{+} \pi^{-}\right]_{D} K^{ \pm}$and $B^{ \pm} \rightarrow\left[\pi^{ \pm} K^{\mp} \pi^{+} \pi^{-}\right]_{D} \pi^{ \pm}$with a significance of 5.1 and $>10 \sigma$, respectively. Details are discussed in Ref. [23].

\subsection{Combination of $B^{ \pm} \rightarrow D h^{ \pm}$analyses}

The results presented in the previous sections have been used to perform a combined measurement for the CKM phase $\gamma$. Information about strong phase differences in $D \rightarrow K^{-} \pi^{+}$and $D \rightarrow K^{-} \pi^{+} \pi^{+} \pi^{-}$decays from CLEO [24] and evidence for a difference of the $C P$ asymmetries in $D \rightarrow K K$ and $D \rightarrow \pi \pi$ decays [25-29] is also used. For more information see Ref. [30].

From the previously discussed $B^{ \pm} \rightarrow D K$ results, we find an unambiguous best-fit value in $\left[\begin{array}{ll}0 & 18,0\end{array}\right]^{\circ}$ of

$$
\gamma=\left(71.1_{-15.7}^{+16.6}\right)^{\circ}
$$

using a frequentist statistical procedure. The result suggests very good prospects for a measurement based on the full dataset. Additional $\gamma$-sensitive measurements will also be included in the future. With the data currently available on tape, $\mathrm{LHCb}$ should be able to reduce the error quoted above by at least a factor of two.

\section{Search for rare $B$ decays}

Rare heavy flavour decays provide stringent tests of the Standard Model of particle physics and allow to test for possible new physics scenarios. $\mathrm{LHCb}$ is the ideal place for these searches as it has recorded the worlds largest sample of beauty hadrons.

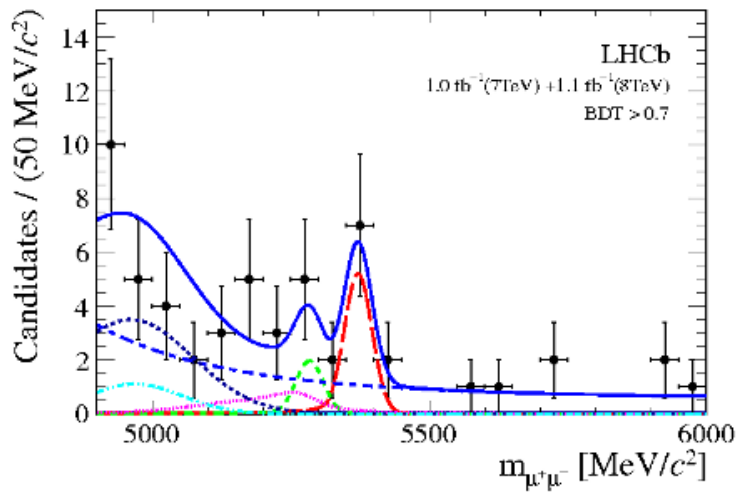

Figure 1. Dimuon invariant mass distribution of $B_{S}^{0} \rightarrow \mu^{+} \mu^{-}$candidates with signal-like BDT responses. The different fit components are: $B_{S}^{0} \rightarrow \mu^{+} \mu^{-}$(red long dashed), $B^{0} \rightarrow \mu^{+} \mu^{-}$(green medium dashed), $B_{(s)}^{0} \rightarrow h^{+} h^{-}$(pink dotted), $B^{0} \rightarrow \pi^{-} \mu^{+} v_{\mu}$ (blue short dashed) and $B^{0(+)} \rightarrow \pi^{0(+)} \mu^{+} \mu^{-}$(light blue dot dashed), and the combinatorial background (blue medium dashed).

Rare processes that proceed via Flavor Changing Neutral Currents (FCNC) are forbidden at tree level in the SM. They can proceed via loop level electroweak $\left(Z^{0}, \gamma\right)$ penguin or box diagrams. In extensions to the SM, new virtual particles can enter at loop level, modifying the amplitude of the process or the Lorentz structure of the decay vertex. Possible deviations from the SM predictions on branching fractions, angular distributions and asymmetries could lead to the discovery of physics beyond the SM.

\subsection{Searches for $B_{S}^{0} \rightarrow \mu^{+} \mu^{-}$and $B^{0} \rightarrow \mu^{+} \mu^{-}$decays}

The branching fraction of the decays $B_{s}^{0} \rightarrow \mu^{+} \mu^{-}$and $B^{0} \rightarrow \mu^{+} \mu^{-}$are suppressed in the SM. Precise predictions of their branching fractions, $\mathcal{B}\left(B_{s}^{0} \rightarrow \mu^{+} \mu^{-}\right)=(3.23 \pm$ $0.27) \times 10^{-9}$ and $\mathcal{B}\left(B^{0} \rightarrow \mu^{+} \mu^{-}\right)=(1.07 \pm 0.10) \times 10^{-10}[31]$ make these modes powerful probes in the search for deviations from the SM, especially in models with a nonstandard Higgs sector. Taking the measured finite width difference of the $B_{s}^{0}$ system into account [32], the time integrated branching fraction of $B_{S}^{0} \rightarrow \mu^{+} \mu^{-}$that should be compared to the experimental value is $(3.54 \pm 0.30) \times 10^{-9}$.

Previous searches already constrain possible deviations from the SM predictions. The lowest previously published limits are $\mathcal{B}\left(B_{s}^{0} \rightarrow \mu^{+} \mu^{-}\right)<4.5 \times 10^{-9}$ and $\mathcal{B}\left(B^{0} \rightarrow \mu^{+} \mu^{-}\right)<1.0 \times 10^{-9}$ at $95 \%$ confidence level from $\mathrm{LHCb}$, using $1.0 \mathrm{fb}^{-1}$ of data collected in $p p$ collisions in 2011 at $\sqrt{s}=7 \mathrm{TeV}$ [33]. This article refers to an update of this search with an additional $1.1 \mathrm{fb}^{-1}$ of data recorded in 2012 at $\sqrt{s}=8 \mathrm{TeV}$. The analysis procedure is described in detail in Ref. [34].

In $\mathrm{LHCb}, B_{S}^{0} \rightarrow \mu^{+} \mu^{-}$and $B^{0} \rightarrow \mu^{+} \mu^{-}$candidates are selected by requiring two high quality muon candidates displaced with respect to any $p p$ interaction vertex and forming a secondary vertex. They are then classified using a Boosted Decision Tree (BDT) based on the kinematic properties of the reconstructed $B_{S}^{0}$ and $B^{0}$ candidate. 
The dimuon invariant mass of the candidates with a signal-like BDT response is shown in Fig. 1. We observe an excess of $B_{S}^{0} \rightarrow \mu^{+} \mu^{-}$candidates with respect to the background expectation. The probability that the background could produce such an excess is $5.3 \times 10^{-4}$, corresponding to a signal significance of $3.5 \sigma$ and therefore providing first evidence for the $B_{s}^{0} \rightarrow \mu^{+} \mu^{-}$decay. A maximum-likelihood fit gives a branching fraction of $\mathcal{B}\left(B_{S}^{0} \rightarrow \mu^{+} \mu^{-}\right)=\left(3.2_{-1.2}^{+1.5}\right) \times 10^{-9}$, where the statistical uncertainty is $95 \%$ of the total uncertainty. This result is clearly in agreement with the Standard Model expectation. The observed number of $B^{0} \rightarrow \mu^{+} \mu^{-}$candidates is consistent with the background expectation, giving an upper limit of $\mathcal{B}\left(B^{0} \rightarrow \mu^{+} \mu^{-}\right)<9.4 \times 10^{-10}$ at $95 \%$ confidence level.

\subsection{Isospin asymmetry in $B \rightarrow K^{(*)} \mu^{+} \mu^{-}$decays}

The FCNC decays $B \rightarrow K^{(*)} \mu^{+} \mu^{-}$are forbidden at tree level in the SM and must proceed via loop or box diagrams. Predictions for the branching fractions of these decays suffer from relatively large uncertainties due to form factor estimates. However, theoretically clean observables can be constructed from ratios or asymmetries where the leading form factor uncertainties cancel. The $C P$ averaged isospin asymmetry $\left(A_{\mathrm{I}}\right)$ is such an observable. It is defined as

$$
A_{\mathrm{I}}=\frac{\mathcal{B}\left(B^{0} \rightarrow K^{(*) 0} \mu^{+} \mu^{-}\right)-\frac{\tau_{0}}{\tau_{+}} \mathcal{B}\left(B^{+} \rightarrow K^{(*)+} \mu^{+} \mu^{-}\right)}{\mathcal{B}\left(B^{0} \rightarrow K^{(*) 0} \mu^{+} \mu^{-}\right)+\frac{\tau_{0}}{\tau_{+}} \mathcal{B}\left(B^{+} \rightarrow K^{(*)+} \mu^{+} \mu^{-}\right)},
$$

where $\mathcal{B}(B \rightarrow f)$ is the branching fraction of the $B \rightarrow f$ decay and $\tau_{0} / \tau_{+}$is the ratio of the lifetimes of the $B^{0}$ and $B^{+}$mesons. For $B \rightarrow K^{*} \mu^{+} \mu^{-}$, the $\mathrm{SM}$ prediction for $A_{\mathrm{I}}$ is around $-1 \%$ in the di-muon mass squared $\left(q^{2}\right)$ region below the $J / \psi$ resonance, apart from the very low $q^{2}$ region where it rises to $O(10 \%)$ as $q^{2}$ approaches zero [35].

The isospin asymmetries are determined by measuring the differential branching fractions of $B^{+} \rightarrow K^{+} \mu^{+} \mu^{-}, B^{0} \rightarrow K_{s}^{0} \mu^{+} \mu^{-}, B^{0} \rightarrow\left(K^{* 0} \rightarrow K^{+} \pi^{-}\right) \mu^{+} \mu^{-}$ and $B^{+} \rightarrow\left(K^{*+} \rightarrow K_{s}^{0} \pi^{+}\right) \mu^{+} \mu^{-}$. The $K_{s}^{0}$ meson is reconstructed via the $K_{S}^{0} \rightarrow \pi^{+} \pi^{-}$decay mode. In the 2011 data sample of $1 \mathrm{fb}^{-1}$ LHCb has been able to select about 60 $B^{0} \rightarrow K_{s}^{0} \mu^{+} \mu^{-}$decays, reporting an observation at $5.7 \sigma$ for this decay [36], and about $80 B^{0} \rightarrow\left(K^{* 0} \rightarrow K^{+} \pi^{-}\right) \mu^{+} \mu^{-}$ decays, which are comparable in size to the samples available for these modes in the full data sets of the $B$-factories.

The isospin asymmetries as a function of $q^{2}$ for $B \rightarrow$ $K \mu^{+} \mu^{-}$and $B \rightarrow K^{*} \mu^{+} \mu^{-}$are shown in Fig. 2. The two $q^{2}$ bins below $4.3 \mathrm{GeV} / c^{2}$ and the highest bin above $16 \mathrm{GeV} / c^{2}$ have the most negative isospin asymmetry in the $B \rightarrow K \mu^{+} \mu^{-}$channel. These $q^{2}$ regions are furthest from the charmonium regions and are therefore cleanly predicted theoretically. This asymmetry is dominated by a deficit in the observed $B^{0} \rightarrow K^{0} \mu^{+} \mu^{-}$signal. Ignoring the small correlation of errors between each $q^{2}$ bin, the significance of the deviation from zero integrated across $q^{2}$ is calculated to be $4.4 \sigma$. The $B \rightarrow K^{*} \mu^{+} \mu^{-}$case agrees with the SM prediction of almost zero isospin asymmetry [35]. All results agree with previous measurements [37-39].
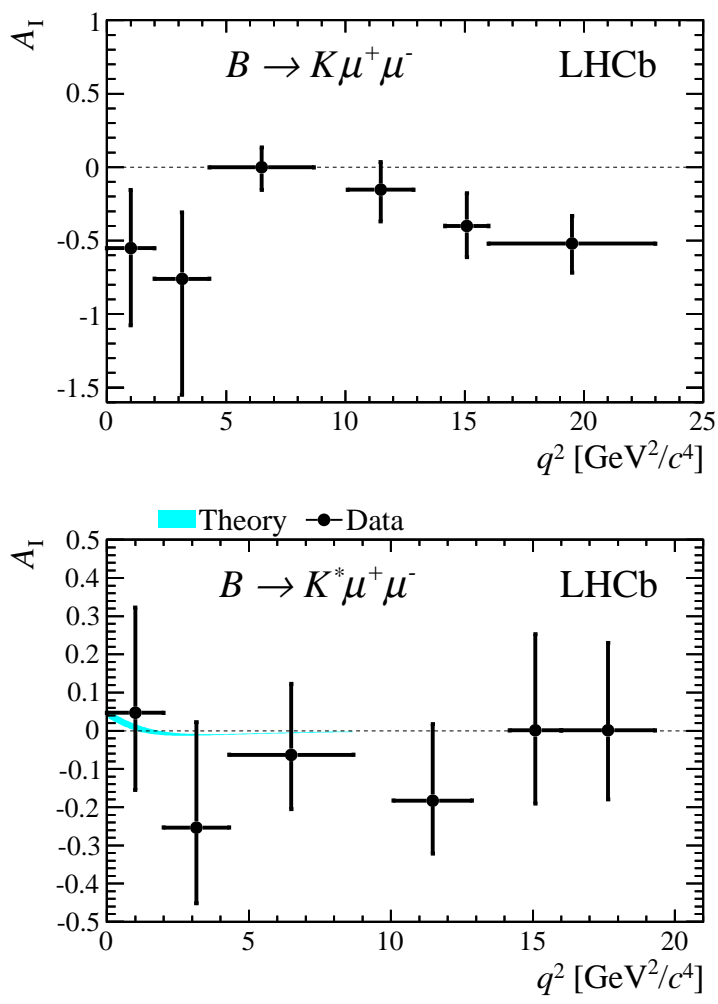

Figure 2. Isospin asymmetry of (top) $B \rightarrow K \mu^{+} \mu^{-}$and (bottom) $B \rightarrow K^{*} \mu^{+} \mu^{-}$. For $B \rightarrow K^{*} \mu^{+} \mu^{-}$the theoretical SM prediction, which is very close to zero, is shown for $q^{2}$ below $8.68 \mathrm{GeV} / c^{2}$.

\section{Observation of $D^{0}-\bar{D}^{0}$ mixing}

The mixing of neutral mesons is a powerful probe to discover physics beyond the standard model. Oscillations have been observed in the $K^{0}-\bar{K}^{0}[40], B^{0}-\bar{B}^{0}[41]$ and $B_{s}^{0}-\bar{B}_{s}^{0}[42]$ systems, all with rates in agreement with SM expectations. Evidence of $D^{0}-\bar{D}^{0}$ oscillations has been reported by three experiments using different $D^{0}$ decay channels [43-47]. However, only the combination of these measurements provided so far confirmation of $D^{0}-\bar{D}^{0}$ oscillations, also referred to as charm mixing, with more than $5 \sigma$ significance [29].

The search for $D^{0}-\bar{D}^{0}$ oscillations has been performed by studying the time-dependent ratio of $D^{0} \rightarrow K^{+} \pi^{-}$to $D^{0} \rightarrow K^{-} \pi^{+}$decay rates in $D^{*+}$-tagged events. ${ }^{1}$ In the following this measurement is summarized, using $1.0 \mathrm{fb}^{-1}$ of integrated luminosity recorded by the $\mathrm{LHCb}$ experiment in 2011. Details about the analysis can be found in Ref. [48]. The $D^{0}$ flavour at production time is determined using the charge of the soft (low-momentum) pion, $\pi_{s}^{+}$, in the strong $D^{*+} \rightarrow D^{0} \pi_{s}^{+}$decay. The $D^{*+} \rightarrow D^{0}\left(\rightarrow K^{-} \pi^{+}\right) \pi_{s}^{+}$process is referred to as right-sign (RS), whereas the $D^{*+} \rightarrow$ $D^{0}\left(\rightarrow K^{+} \pi^{-}\right) \pi_{S}^{+}$is designated as wrong-sign (WS). The $\mathrm{RS}$ process is dominated by a Cabibbo-favoured (CF) decay amplitude, whereas the WS amplitude includes contributions from both the doubly-Cabibbo-suppressed (DCS) $D^{0} \rightarrow K^{+} \pi^{-}$decay, as well as $D^{0}-\bar{D}^{0}$ mixing followed

\footnotetext{
${ }^{1}$ The inclusion of charge-conjugated modes is implied throughout this section.
} 


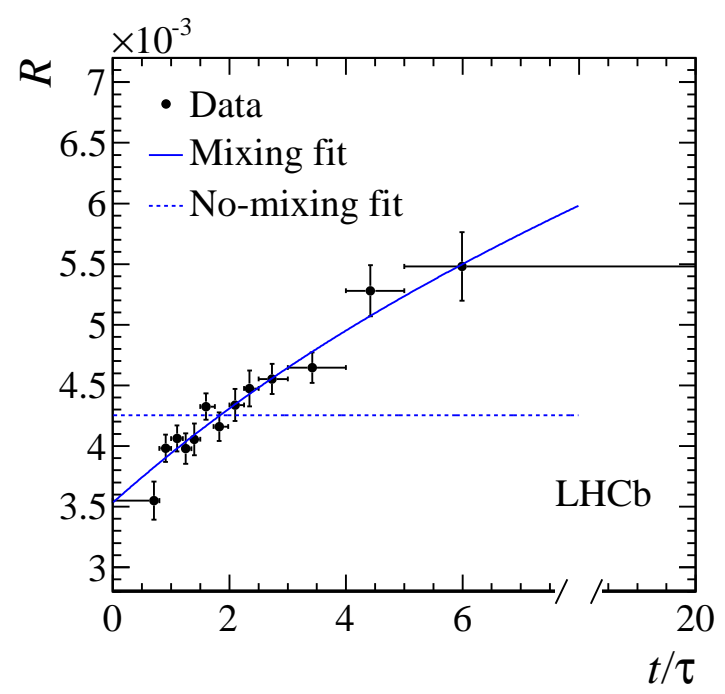

Figure 3. Decay-time evolution of the ratio, $R$, of WS $D^{0} \rightarrow$ $K^{+} \pi^{-}$to RS $D^{0} \rightarrow K^{-} \pi^{+}$yields (points) with the projection of the mixing allowed (solid line) and no-mixing (dashed line) fits overlaid.

Table 1. Results of the time-dependent fit to the data. The uncertainties include statistical and systematic sources; ndf indicates the number of degrees of freedom.

\begin{tabular}{lcc}
\hline Fit type $\left(\chi^{2} / \mathrm{ndf}\right)$ & Parameter & Fit result $\left(10^{-3}\right)$ \\
\hline Mixing $(9.5 / 10)$ & $R_{D}$ & $3.52 \pm 0.15$ \\
& $y^{\prime}$ & $7.2 \pm 2.4$ \\
& $x^{\prime 2}$ & $-0.09 \pm 0.13$ \\
\hline No mixing $(98.1 / 12)$ & $R_{D}$ & $4.25 \pm 0.04$ \\
\hline
\end{tabular}

by the favored $\bar{D}^{0} \rightarrow K^{+} \pi^{-}$decay. In the limit of small mixing, and assuming negligible $C P$ violation, the timedependent ratio, $R$, of WS to RS decay rates is approximated by

$$
R(t) \approx R_{D}+\sqrt{R_{D}} y^{\prime} \frac{t}{\tau}+\frac{x^{\prime 2}+y^{\prime 2}}{4}\left(\frac{t}{\tau}\right)^{2},
$$

where $t / \tau$ is the decay time expressed in units of the average $D^{0}$ lifetime $\tau, R_{D}$ is the ratio of DCS to CF decay rates, $x^{\prime}=x \cos \delta+y \sin \delta, y^{\prime}=y \cos \delta-x \sin \delta$, and $\delta$ is the strong phase difference between the DCS and CF amplitudes.

We reconstruct approximately $3.6 \times 10^{4} \mathrm{WS}$ and $8.4 \times$ $10^{6} \mathrm{RS}$ decays. To determine the time-dependent WS/RS ratio the data are divided into thirteen $D^{0}$ decay time bins, chosen to have a similar number of candidates in each bin.

The fit to the decay-time evolution of the WS /RS ratio is shown in Fig. 3 (solid line), with the values and uncertainties of the parameters $R_{D}, y^{\prime}$ and $x^{\prime 2}$ listed in Table 1. The value of $x^{\prime 2}$ is found to be negative, but consistent with zero. As the dominant systematic uncertainties are treated within the fit procedure (all other systematic effects are negligible), the quoted errors account for systematic as well as statistical uncertainties. When the systematic biases are not included in the fit, the estimated uncertainties on $R_{D}, y^{\prime}$ and $x^{\prime 2}$ become respectively $6 \%, 10 \%$ and $11 \%$

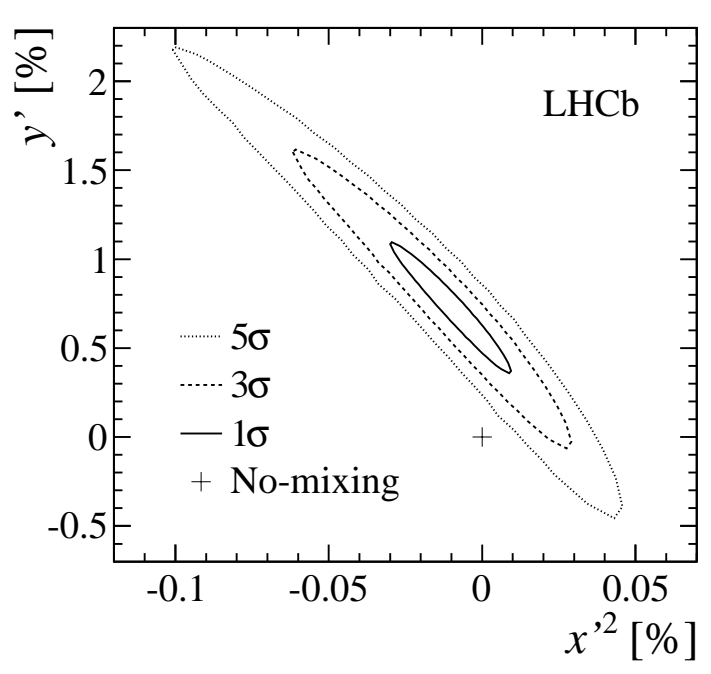

Figure 4. Estimated confidence-level (CL) regions in the $\left(x^{\prime 2}, y^{\prime}\right)$ plane for $1-\mathrm{CL}=0.317(1 \sigma), 2.7 \times 10^{-3}(3 \sigma)$ and $5.73 \times 10^{-7}$ $(5 \sigma)$. Systematic uncertainties are included. The cross indicates the no-mixing point.

smaller, showing that the quoted uncertainties are dominated by their statistical component. To evaluate the significance of this mixing result we determine the change in the fit $\chi^{2}$ when the data are described under the assumption of the no-mixing hypothesis (dashed line in Fig. 3). Under the assumption that the $\chi^{2}$ difference, $\Delta \chi^{2}$, follows a $\chi^{2}$ distribution for two degrees of freedom, $\Delta \chi^{2}=88.6$ corresponds to a $p$-value of $5.7 \times 10^{-20}$, which excludes the no-mixing hypothesis at 9.1 standard deviations. This is illustrated in Fig. 4 where the $1 \sigma, 3 \sigma$ and $5 \sigma$ confidence regions for $x^{\prime 2}$ and $y^{\prime}$ are shown. The measured values of the mixing parameters are compatible with and have substantially better precision than those from previous measurements [43, 45, 49].

\section{Conclusion}

LHCb performed a first measurement of the weak phase $\gamma$ from decays at tree level, using $1.0 \mathrm{fb}^{-1}$ of data collected in 2011. The obtained result of $\gamma=\left(71.1_{-15.7}^{+16.6}\right)^{\circ}$ is already of comparable precision as the world average based on data from the $B$-factories and the Tevatron.

The search for the rare decay $B_{s}^{0} \rightarrow \mu^{+} \mu^{-}$with $1.0 \mathrm{fb}^{-1}$ and $1.1 \mathrm{fb}^{-1}$ of $p p$ collision data collected at $\sqrt{s}=7 \mathrm{TeV}$ and $\sqrt{s}=8 \mathrm{TeV}$, respectively, shows an excess of events with respect to the background-only prediction with a statistical significance of 3.5 . This is the first evidence for the decay $B_{S}^{0} \rightarrow \mu^{+} \mu^{-}$. The measured branching fraction $\mathcal{B}\left(B_{S}^{0} \rightarrow \mu^{+} \mu^{-}\right)=\left(3.2_{-1.2}^{+1.5}\right) \times 10^{-9}$ is in agreement with the SM prediction.

The large charm production cross-section at the LHC provided a copious data sample for a measurement of $D^{0}-\bar{D}^{0}$ mixing. The decay time dependence of the ratio between $D^{0} \rightarrow K^{+} \pi^{-}$and $D^{0} \rightarrow K^{-} \pi^{+}$decays exclude the no-mixing hypothesis at $9.1 \sigma$. This is the first observation of $D^{0}-\bar{D}^{0}$ oscillations in a single measurement. 
During the 2012 run at $\sqrt{s}=8 \mathrm{TeV}, 2.1 \mathrm{fb}^{-1}$ of data were collected, which will allow $\mathrm{LHCb}$ to increase the precision on the results presented here and to pursue the search for physics beyond the Standard Model.

\section{References}

[1] O. Callot, LHCb detector status and upgrade, these proceedings.

[2] N. Cabibbo, Phys. Rev. Lett. 10 (1963) 531.

[3] M. Kobayashi and T. Maskawa, Prog. Theor. Phys. 49 (1973) 652.

[4] CKMfitter group, J. Charles et al., Eur. Phys. J. C41 (2005) 028, arXiv:hep-ph/0406184. Numbers from: http://ckmfitter.in2p3.fr/www/results/plots_moriond12.

[5] UTfit collaboration, M. Bona et al., JHEP $\mathbf{5 0 7}$ (2005) 1, arXiv:hep-ph/0501199. Numbers taken from: http://www.utfit.org/UTfit /ResultsSummer2012PreICHE

[6] M. Gronau and D. London, Phys. Lett. B253 (1991) 483.

[7] M. Gronau and D. Wyler, Phys. Lett. B265 (1991) 172.

[8] D. Atwood, I. Dunietz, and A. Soni, Phys. Rev. Lett. 78 (1997) 3257, arXiv:hep-ph/9612433.

[9] D. Atwood, I. Dunietz, and A. Soni, Phys. Rev. D63 (2001) 036005, arXiv:hep-ph/0008090.

[10] A. Giri, Y. Grossman, A. Soffer, and J. Zupan, Phys. Rev. D68 (2003) 054018, arXiv:hep-ph/0303187.

[11] BaBar collaboration, B. Aubert et al., Phys. Rev. Lett. 95 (2005) 121802, arXiv:hep-ex/0504039.

[12] BaBar collaboration, B. Aubert et al., Phys. Rev. D78 (2008) 034023, arXiv:0804.2089.

[13] BaBar collaboration, P. del Amo Sanchez et al., Phys. Rev. Lett. 105 (2010) 121801, arXiv:1005.1096.

[14] Belle collaboration, A. Poluektov et al., Phys. Rev. D70 (2004) 072003, arXiv:hep-ex/0406067.

[15] Belle collaboration, A. Poluektov et al.,

Phys. Rev. D73 (2006) 112009, arXiv:hep-ex/0604054.

[16] Belle collaboration, A. Poluektov et al., Phys. Rev. D81 (2010) 112002, arXiv:1003.3360.

[17] CLEO collaboration, J. Libby et al., Phys. Rev. D82 (2010) 112006, arXiv:1010.2817.

[18] A. Bondar and A. Poluektov, Eur. Phys. J. C47 (2006) 347, arXiv:hep-ph/0510246.

[19] Heavy Flavor Averaging Group, D.Asner et al., arXiv:1010.1589, updates available online at http://www.slac.stanford.edu/xorg/hfag.

[20] LHCb collaboration, R. Aaij et al., Phys. Lett. B712 (2012) 203, arXiv:1203.3662.

[21] Particle Data Group, Beringer, J. et al., Phys. Rev. D86 (2012) 010001.

[22] LHCb collaboration, R. Aaij et al., Phys. Lett. B718 (2012) 43, arXiv:1209.5869.
[23] LHCb collaboration, R. Aaij et al., LHCb-CONF-2012-030 (2012).

[24] CLEO collaboration, N. Lowrey et al., Phys. Rev. D80 (2009) 031105, arXiv:0903.4853.

[25] BaBar collaboration, Aubert, et al., Phys. Rev. Lett. 100 (2008) 061803, arXiv:0709.2715.

[26] Belle collaboration, M. Staric et al., Phys. Lett. B670 (2008) 190, arXiv:0807.0148.

[27] LHCb collabortion, R. Aaij et al., Phys. Rev. Lett.108 (2012) 111602, arXiv:1112.0938.

[28] CDF collabortion, T. Aaltonen et al., Phys. Rev. Lett.109 (2012) 111801, arXiv:1207.2158.

[29] Heavy Flavor Averaging Group, Y.Amhis et al., arXiv:1207.1158, updates available online at http://www.slac.stanford.edu/xorg/hfag.

[30] LHCb collaboration, R. Aaij et al., LHCb-CONF-2012-032 (2012).

[31] A. J. Buras et al., Eur. Phys. J. C72 (2012) 2172, arXiv:1208.0934.

[32] K. de Bruyn et al., Phys. Rev. Lett. 109 (2012) 041801, arXiv:1204.1737.

[33] LHCb collaboration, R. Aaij et al., Phys. Rev. Lett. 108 (2012) 231801, arXiv:1203.4493.

[34] LHCb collaboration, R. Aaij et al., Phys. Rev. Lett. 110 (2013) 021801, arXiv:1211.2674.

[35] T. Feldmann amd J. Matias, JHEP 0301 (2003) 0301, arXiv:hep-ph/0212158.

[36] LHCb collaboration, R. Aaij et al., JHEP 07 (2012) 133, arXiv:1205.3422.

[37] Belle collaboration, J.-T. Wei et al., Phys. Rev. Lett. 103 (2009) 171801, arXiv:0904.0770.

[38] BaBar collaboration, J.P. Lees et al., submitted to Phys. Rev. D, arXiv:1204.3933.

[39] CDF collaboration, T. Aaltonen et al., Phys. Rev. Lett. 107 (2011) 201802, arXiv:1107.3753.

[40] K. Lande et al., Phys. Rev. bf 103 (1956) 1901.

[41] ARGUS collaboration, H. Albrecht et al., Phys. Lett. B 192 (1987) 245.

[42] CDF collaboration, A. Abulencia et al., Phys. Rev. Lett. 97 (2006) 242003, arXiv:hep-ex/0609040.

[43] BaBar collaboration, B. Aubert et al., Phys. Rev. Lett. 98 (2007) 211802, arXiv:hep-ex/0703020.

[44] Belle collaboration, M. Staric et al., Phys. Rev. Lett. 98 (2007) 211803, arXiv:hep-ex/0703036.

[45] CDF collaboration, T. Aaltonen et al., Phys. Rev. Lett. 100 (2008) 121802, arXiv:0712.1567.

[46] BaBar collaboration, B. Aubert et al., Phys. Rev. Lett. 103 (2009) 211801, arXiv:0807.4544.

[47] BaBar collaboration, B. Aubert et al., Phys. Rev. D 80 (2009) 071103, arXiv:0908.0761.

[48] LHCb collaboration, R. Aaij et al., Phys. Lett. B712 (2012) 203, arXiv:1211.1230.

[49] Belle collaboration, L.M. Zhang et al., Phys. Rev. Lett. 96 (2006) 151801, arXiv:hep-ex/0601029. 